\title{
Human Dental Pulp Stem Cells Differentiate into Oligodendrocyte Progenitors Using the Expression of Olig2 Transcription Factor
}

\author{
Nahid Askaria ${ }^{a}$ Mohammad Mehdi Yaghoobi ${ }^{\mathrm{b}} \quad$ Mehdi Shamsara $^{\mathrm{a}}$ \\ Saeed Esmaeili-Mahani ${ }^{c}$ \\ ${ }^{a}$ National Institute of Genetic Engineering and Biotechnology, Tehran, ${ }^{b}$ Department of Biotechnology, Institute \\ of Science and High Technology and Environmental Sciences, Graduate University of Advanced Technology, and \\ 'Department of Biology, Faculty of Sciences, Shahid Bahonar University of Kerman, Kerman, Iran
}

\section{Key Words}

Dental pulp stem cells - Olig2 transcription factor .

Oligodendrocyte progenitors · Transfection

\begin{abstract}
The helix-loop-helix transcription factor Olig2 is essential for lineage determination of oligodendrocytes. Differentiation of stem cells into oligodendrocytes and transplanting them is a novel strategy for the repair of different demyelination diseases. Dental pulp stem cells (DPSCs) are of great interest in regenerative medicine due to their potential for repairing damaged tissues. In this study, DPSCs were isolated from human third molars and transfected with the human Olig2 gene as a differentiation inducer for the oligodendrogenic pathway. Following the differentiation procedure, the expression of Sox2, NG2, PDGFRa, Nestin, MBP, Olig2, Oct4, glial fibrillary acidic protein and $\mathrm{A} 2 \mathrm{~B} 5$ as stage-specific markers was studied by real-time RT-qPCR, immunocytochemistry and Western blot analysis. The cells were transplanted into a mouse model of local sciatic damage by lysolecithin as a model for demyelination. Oligodendrocyte progenitor cells (OPCs) actively remyelinated and recovered the lysolecithin-
\end{abstract}

induced damages in the sciatic nerve as revealed by treadmill exercise, the von Frey filament test and hind paw withdrawal in response to a thermal stimulus. Recovery of behavioral reflexes occurred 2-6 weeks after OPC transplantation. The results demonstrate that the expression of Olig2 in DPSCs reduces the expression of stem cell markers and induces the development of oligodendrocyte progenitors as revealed by the emergence of oligodendrocyte markers. DPSCs could be programmed into oligodendrocyte progenitors and considered as a simple and valuable source for the cell therapy of neurodegenerative diseases.

(c) 2015 S. Karger AG, Basel

\begin{tabular}{ll}
\hline Abbreviations used in this paper \\
\hline CNS & central nervous system \\
DPSCs & dental pulp stem cells \\
GFAP & glial fibrillary acidic protein \\
OPCs & oligodendrocyte progenitor cells \\
PBS & phosphate-buffered saline \\
PNS & peripheral nervous system \\
\hline
\end{tabular}

\section{KARGER}

E-Mail karger@karger.com www.karger.com/cto
(C) 2015 S. Karger AG, Basel

$1422-6405 / 15 / 2002-0093 \$ 39.50 / 0$
Mohammad Mehdi Yaghoobi

End of Haft Bagh-e-Alavi Highway Knowledge Paradise Kerman 7631133131 (Iran)

E-Mail m.yaghoobi@kgut.ac.ir 


\section{Introduction}

Myelination and remyelination are two critical steps for the function and repair of the nervous system; however, they can be affected by various demyelinating diseases. Although endogenous neural stem cells proliferate following injury, they are inadequate to effect repair, i.e. the nervous system has a limited capacity for repair following degeneration or injury. Both the central nervous system (CNS) and the peripheral nervous system (PNS) are susceptible to demyelinating disorders. Oligodendrocytes are derived from neural stem cells through successive stages of lineage restriction. They are the main cells that form myelin sheaths in the CNS and are equivalent to Schwann cells in the PNS.

Oligodendrocyte progenitor cells (OPCs) exist in the adult CNS and retain an imprint of their developmental origin [Lu et al., 2000; Liu and Rao, 2004; Cai et al., 2005]. Oligodendrocyte lineage transcription factor 2 (Olig2) is a member of the OLIG family of basic helix-loop helix transcription factors and plays an important role in determining the ultimate location of oligodendrocytes and motor neurons during development [Lu et al., 2000; Vallstedt et al., 2005]. Various studies have demonstrated that Olig1 and Olig2 are critically necessary in myelination and in the formation of oligodendrocytes during embryogenesis [Rogister et al., 1999; Grinspan, 2002; Rowitch et al., 2002].

Dental pulp stem cells (DPSCs) can be readily obtained by a noninvasive procedure and have been shown to possess properties similar to mesenchymal stem cells and neural stem cells. They are multipotent stem cells derived from the mesenchyme and neural crest. They exhibit plasticity and multipotential capabilities and multidifferentiation potentials [Yalvac et al., 2009]. Stem cells isolated from the pulp of wisdom teeth have been differentiated into dentin-producer odontoblasts, adipocytes, osteoblasts, skeletal and/or smooth-muscle cells, neural cells, elastic cartilage cells and endothelial cells both in vitro and in vivo [Tatullo et al., 2014].

The intention of this study was to develop an alternative strategy to preserve and maximize the potential application of DPSCs for repairing demyelination or other injury in the CNS. Here, we show that Olig2 can promote oligodendrocyte differentiation in DPSCs and that it regulates the growth of normal OPCs. To determine the proficiency of these cells in remyelination, we used a selective demyelinating agent, lysolecithin [Hall and Gregson, 1971], on the mouse sciatic nerve as an example of a peripheral nerve. We analyzed the remyelination and their recovery by DPSC-derived OPCs.

\section{Materials and Methods}

\section{Human Dental Pulp Stem Cell Culture}

Human DPSCs were cultured according to Ebrahimi et al. [2011]. Briefly, wisdom teeth without caries were removed from 5 healthy young adults (aged 20-30 years) after obtaining informed consent. The teeth were then immediately immersed in precooled, phosphate-buffered saline (PBS) containing penicillin $(200 \mathrm{U} / \mathrm{ml})$ and streptomycin $(200 \mathrm{mg} / \mathrm{ml})$ and transported to the cell culture laboratory. In a laminar flow cabinet, the teeth were then disinfected with povidone-iodine for approximately $5 \mathrm{~min}$. PBS was used to wash the teeth repeatedly. The dental crown was cracked, and the pulp was removed. The dental pulp tissue was minced into small pieces with a scalpel and transferred to centrifuge tubes containing $3 \mathrm{mg} / \mathrm{ml}$ collagenase/dispase. After digestion at $37^{\circ} \mathrm{C}$ for $20-30 \mathrm{~min}$, the solution was mixed to form a single-cell suspension. The cell suspension was centrifuged at $1,200 \mathrm{rpm}$ for $5 \mathrm{~min}$, and the supernatant was removed. Cell suspensions were seeded in $60-\mathrm{mm}$ culture dishes containing minimum essential medium (alpha modification) with 10\% FBS, 100 $\mathrm{U} / \mathrm{ml}$ penicillin $\mathrm{G}, 100 \mu \mathrm{g} / \mathrm{ml}$ streptomycin and $1 \mu \mathrm{g} / \mathrm{ml}$ amphotericin $\mathrm{B}$. The pulp segments were separated from the cells and fixed under a coverslip and cultured in the same medium. All samples were incubated at $37^{\circ} \mathrm{C}$ in a $5 \% \mathrm{CO}_{2}$ incubator, and the medium was replaced every 2 days. The pulp tissues and sprouted cells were observed under an inverted microscope (Axiovert 200, Carl Zeiss) daily. Colony formation was observed 10-14 days thereafter. The cells were passaged 1:4 when they were $80-90 \%$ confluent with $0.25 \%$ trypsin/ $1 \mathrm{~mm}$ EDTA. The cell viability was determined by trypan blue staining. Cells were cryopreserved in minimum essential medium (alpha modification) containing 30\% FBS and 5\% DMSO and stored in liquid nitrogen. The cells were thawed rapidly for reseeding and their viability was determined again by trypan blue staining. They were cultured on coverslips, fixed with methanol-acetone and stained with $1 \mathrm{mg} / \mathrm{ml}$ Hoechst 33258 to rule out the presence of mycoplasma parasites inside them.

\section{Gene Cloning and Transfection}

The entire coding region of the human Olig2 gene (972 bp) was directly synthesized and cloned in pUC57 plasmid by gene synthesis (GenScript, USA). As the NheI and EcoRV restriction sites were incorporated at the beginning and the end of the open reading frame (ORF), respectively, it was subsequently subcloned into the pcDNA3.1(+) (Invitrogen, USA) expression vector. The pcDNA3.1 containing the Olig2 ORF (pcDNA-Olig2) was transformed into the DH5a strain of E. coli and was extracted using the Qiagen plasmid midi kit according to the manufacturer's instructions. Sequence analysis (BIONEER, Korea) revealed the correct size and orientation of the Olig2 gene (data not shown).

X-tremeGENE HP DNA transfection reagent (Roche, Switzerland) was used for transfection of the DPSCs according to the manufacturer's instructions.

DPSCs at passage 3-5 were cultured in seven 60-mm dishes until the cell density reached $70-80 \%$ confluence. Just before transfection, the medium was replaced with minimum essential medium (alpha modification) without FBS and antibiotics. The transfection reagent $(1,2,3$ and $4 \mu \mathrm{l})$ and pcDNA-Olig2 $(2 \mu \mathrm{g})$ were added to a tube, mixed gently and incubated for $15 \mathrm{~min}$ at room temperature. The mixture was applied drop-wise directly to the medium. One
Askari/Yaghoobi/Shamsara/ Esmaeili-Mahani 
Table 1. Primer sequences and product sizes used for RT-qPCR

\begin{tabular}{|c|c|c|c|}
\hline Gene name & RefSeq No. & Primer sequence & Product size, bp \\
\hline$\beta$-actin & NM_001101 & $\begin{array}{l}\text { F: GGA CTT CGA GCA AGA GAT GG } \\
\text { R: GAC AGC ACT GTG TTG GCG TA }\end{array}$ & 237 \\
\hline Olig2 & NM_005806.3 & $\begin{array}{l}\text { F: GCT GCG TCT CAA GAT CAA C } \\
\text { R: AGT CGC TTC ATC TCC TCC A }\end{array}$ & 192 \\
\hline$P D G F R \alpha$ & NM_006206 & $\begin{array}{l}\text { F: GTG GGA CAT TCA TTG CGG A } \\
\text { R: AAG CTG GCA GAG GAT TAG G }\end{array}$ & 124 \\
\hline Sox 2 & NM_003106 & $\begin{array}{l}\text { F: ATC AGG AGT TGT CAA GGC AGA G } \\
\text { R: CGC CGC CGA TGA TTG TTA TT }\end{array}$ & 172 \\
\hline Oct4 (POU5F1) & NM_002701.4 & $\begin{array}{l}\text { F: GAC AGG GGG AGG GGA GGA GCT AGG } \\
\text { R: CTT CCC TCC AAC CAG TTG CCC CAA AC }\end{array}$ & 144 \\
\hline Nestin & NM_006617.1 & $\begin{array}{l}\text { F: GAG AAA CAG GGC CTA CAG AG } \\
\text { R: GCT GAG GGA AGT CTT GGA G }\end{array}$ & 168 \\
\hline NG2 & NM_001897.4 & $\begin{array}{l}\text { F: GCT GTG TGG TGT TTG TGT C } \\
\text { R: CAG GAG GTG GAA GTT CAG AG }\end{array}$ & 146 \\
\hline
\end{tabular}

dish was transfected with pcDNA3.1 plasmid backbone without any insertion, to reveal any off-target effects. The other dish was treated with X-tremeGENE HP DNA transfection reagent only and the last dish was treated with plasmid DNA only to reveal any side effects or cytotoxicity. Following transfection, the cells were incubated at $37^{\circ} \mathrm{C}$ in a $5 \% \mathrm{CO}_{2}$ incubator for $48 \mathrm{~h}$ and the medium was replaced with fresh medium supplemented with $500 \mu \mathrm{g} / \mathrm{ml}$ of neomycin as a selection marker. The antibiotic was applied for $48 \mathrm{~h}$ and then ordinary medium was added to the cells.

\section{RNA Extraction and Real-Time qPCR}

Total RNA was extracted from the cells using RNX-plus ${ }^{\mathrm{TM}} \mathrm{Re}-$ agent (Cinnaclon, Iran) according to the manufacturer's protocol. cDNA was synthesized from $1 \mu \mathrm{g}$ of total RNA using M-MuLV-RT and random hexamer primers (Thermo Fisher Scientific Inc.) in a final volume of $20 \mu \mathrm{l}$. Two negative controls without template RNA and M-MuLV-RT enzyme also accompanied each reaction. Real-time RT-qPCR was carried out using the primer sequences listed in table 1 . The reaction was accomplished using $10 \mu \mathrm{l} 2 \mathrm{X}$ FastStart SYBR Green Master ROX (Roche, Germany), $2 \mu \mathrm{l}$ of cDNA and $150 \mathrm{nM}$ of each primer in a $20-\mu \mathrm{l}$ final volume reaction on Rotor Gene 3000 (Corbett Research, Australia). Temperature conditions were as follows: $95^{\circ} \mathrm{C}$ for $4 \mathrm{~min}, 45$ cycles of $95^{\circ} \mathrm{C}$ for $25 \mathrm{~s}, 56-58^{\circ} \mathrm{C}$ (depending on primers) for $25 \mathrm{~s}$ and $72^{\circ} \mathrm{C}$ for $30 \mathrm{~s}$. Melting analysis was induced as follows: $72^{\circ} \mathrm{C}$ for $1 \mathrm{~min}$, followed by $72-99^{\circ} \mathrm{C}$ ramp with 1 -degree increments every $5 \mathrm{~s}$. Data were gathered at the extension step of each cycle and during the melting analysis step on the FAM/SYBR and ROX channels. The collected raw data of the FAM/SYBR channel were first normalized against ROX channel to exclude background fluorescence fluctuation. The data were then exported to an Excel worksheet and analyzed with the LinRegPCR (v2014.2) program [Ruijter et al., 2009].

The initial concentration of every sample was normalized against the $\beta$-actin reference gene concentration to obtain the expression value. Undifferentiated DPSC cDNA templates were se- lected for the calibrator and each experiment was biologically and technically repeated at least 3 times. The paired two-sample t test (Excel, Microsoft Office 2010, USA) was used to compare the gene expression mean at a differentiated time with an undifferentiated time mean. Significance was established at $\mathrm{p}<0.05$.

\section{Immunocytochemistry}

Transfected and untransfected cells cultured on coverslips were fixed with $4 \%$ paraformaldehyde in PBS for $30 \mathrm{~min}$ at $37^{\circ} \mathrm{C}$ and permeabilized with $0.02 \%$ Triton in PBS. The coverslips were then incubated with rabbit anti-human Olig2 and rabbit anti-human glial fibrillary acidic protein (GFAP) primary antibodies (1:100, Sigma) for $24 \mathrm{~h}$ at $4^{\circ} \mathrm{C}$. Next, after washing with PBS 3 times, they were incubated with horseradish peroxidase-conjugated goat anti-rabbit secondary antibody (1:500, Sigma) for $2-4 \mathrm{~h}$ at $4^{\circ} \mathrm{C}$. Diaminobenzidine (Sigma) was used as the substrate for horseradish peroxidase. The primary antibody was excluded for the negative control.

The cells on the coverslips were incubated with 2 other primary antibodies, mouse anti-A2B5 (1:200, Sigma) and rat anti-human MBP (1:300, Sigma) for $24 \mathrm{~h}$ at $4^{\circ} \mathrm{C}$. After washing with PBS 3 times, FITC-conjugated secondary antibodies, goat anti-mouse (1:200, AbD Serotec) and rabbit anti-rat IgG (1:200, Sigma) were applied for $1 \mathrm{~h}$ in the dark at room temperature. After 3 washes with $0.05 \%$ Triton in PBS, the coverslips were mounted with Glycergel $^{\circledR}$ (Dako, Barcelona, Spain) and analyzed under an Axioplan2 fluorescence microscope (Zeiss).

\section{Protein Electrophoresis and Western Blot}

Total protein was extracted using TNE buffer (10 mM Tris- $\mathrm{HCl}$, $\mathrm{pH} 7.8,1 \%$ NP-40, 0.1\% Na-deoxycholate, $150 \mathrm{mM} \mathrm{NaCl}$, $1 \mathrm{mM}$ EDTA, $1 \mathrm{mM}$ PMSF, $1 \mathrm{mg} / \mathrm{ml}$ each of aprotinin, leupeptin and pepstatin, $1 \mathrm{mM} \mathrm{Na} 3 \mathrm{VO} 4$ and $1 \mathrm{mM} \mathrm{NaF}$ ) on ice in the presence of protease inhibitor cocktail (cOmplete, Mini EDTA-free tablets, Roche). From each protein sample, $30 \mu$ were denatured for $5 \mathrm{~min}$ at $100^{\circ} \mathrm{C}$ in the presence of loading buffer $(6.25 \mathrm{mM}$ Tris $\mathrm{pH} 6.8$, 
$12.5 \%$ glycerol, $2.5 \%$ SDS, $0.025 \%$ bromophenol blue and $5 \%$ $\beta$-mercaptoethanol) and separated by $15 \%$ SDS-polyacrylamide gel electrophoresis (10 mg/lane). Separated proteins were transferred at $4^{\circ} \mathrm{C}$ to a nitrocellulose membrane in a wet transference system (Mini Trans-Blot Electrophoretic Transfer Cell, Bio-Rad, Madrid, Spain). The membrane was incubated in TBST buffer $(50 \mathrm{mM}$ Tris, $200 \mathrm{mM}$ $\mathrm{NaCl}$ and $0.1 \%$ Tween-20, pH 7.4), saturated in blocking solution (TBST and 5\% nonfat dry milk) for $30 \mathrm{~min}$ at room temperature and then hybridized overnight at $4^{\circ} \mathrm{C}$ with Olig2 antibody $(1: 100$, Sig$\mathrm{ma})$. The membrane was then washed 3 times with washing solution (TBST) and incubated for $2 \mathrm{~h}$ with horseradish peroxidase-conjugated secondary antibodies (1:500, Sigma). After washing, the membrane was subjected to enhanced chemiluminescence reagents (Santa Cruz Biotechnology, Inc.) and exposed to Kodak X-ray film.

\section{Implantation of DPSC-Derived OPCs in the}

Lysolecithin-Induced, Demyelinated Sciatic Nerves in Mice

Animals

Adult male mice (20-30 g) were obtained from the Kerman University of Medical Sciences, Kerman, Iran. They were maintained in temperature-controlled rooms with a 12-hour/12-hour light/dark cycle and 50\% humidity, with free access to food and water. All experimental procedures were performed in accordance with the principles of laboratory animal care. This research project was approved by the Iran National Science Foundation and High Research Council and relevant committees at the National Institute for Genetic Engineering and Biotechnology of Iran.

\section{Sciatic Nerve Demyelination}

The mice were anesthetized with intraperitoneal injections of $50 \mathrm{mg} / \mathrm{kg}$ ketamine and $5 \mathrm{mg} / \mathrm{kg}$ xylazine. For sciatic nerve demyelination experiments, once the nerve was exposed at mid-thigh level, the two larger fascicles (tibial and peroneal) were separated by blunt dissection from the smallest sural fascicle. Lysolecithin (Sigma) was applied to the tibial and peroneal fascicles for $15 \mathrm{~min}$ [Hall and Gregson, 1971]. Sham control animals were prepared by applying sterile saline.

Preparation of Cells for Transplantation

For tracing cells after transplantation, they were prelabeled with Cell Tracker CM-DiI C7000 (Invitrogen, USA). Before transplantation, the viability of cells was determined by trypan blue.

\section{Cell Transplantation}

Two days prior to the cell transplantation and throughout the experiment, to avoid immunological rejection, mice were immunosuppressed by the addition of $210 \mathrm{mg} / \mathrm{l}$ cyclosporine A (Sandimmune, Novartis Pharmaceuticals, USA). Approximately $2 \times 10^{5}$ cells in $3 \mu \mathrm{l}$ medium were transplanted 1 week after injury using a 5 - $\mu$ l Hamilton syringe. After injection, the cells were allowed to settle for $3 \mathrm{~min}$ before the needle was retracted. The muscle and skin were then sutured and the mice were given appropriate postoperative treatment. The mice were divided into groups: those that received an injection of either OPCs $(n=6)$, PBS $(n=6)$ or DPSCs $(n=6)$ after lysolecithin treatment and a sham group $(n=6)$ that received sterile saline both in the first and second surgeries.

Behavioral Testing

For hind paw withdrawal in response to graded mechanical stimulation, von Frey filament (North Coast Medical, Inc.) was used. It was measured in conscious animals, which provide a pressure against the hairless skin of the hind paws. The threshold response was defined by the filament that caused foot withdrawal at least 3 times during every application. The time for hind paw withdrawal in response to a quantified thermal stimulus was measured using the hotplate. The thermal stimulus was an infrared intensity setting of $50^{\circ} \mathrm{C}$ applied to the mid-plantar surface of each hind paw and the withdrawal was recorded in seconds [Hargreaves et al., 1988]. The treadmill exercise regime consisting of $30 \mathrm{~min}$ of treadmill running at a speed of $12 \mathrm{~m} / \mathrm{min}$ was also used once a week. Baseline measurements were obtained for all animals before surgery. After surgery, behavioral reflex tests were performed in the treated animals and the sham group.

Histochemical Analyses

To study the extent of demyelination, the animals were sacrificed using a lethal dose of anesthesia. The sciatic nerves were immediately stored and postfixed in $4 \%$ paraformaldehyde for $24 \mathrm{~h}$, when some of them were processed and embedded in paraffin. Slices were stained with hematoxylin and eosin (HE). To follow up the fate of the CM-DiI-labeled transplanted cells, after fixation, the sciatic nerves were processed for analysis of the cell implants.

\section{Results}

\section{Isolation and Oligodendrocyte Differentiation of DPSCs}

All steps from the extraction of the teeth up to oligodendrocyte differentiation are illustrated in figure 1. Isolated cells from dental pulp exhibited a high growth rate and were subcultured at 1:5 dilutions every 5-6 days (fig. 2a). They also expressed the stem cell markers Nucleostemin, Oct4, Jmjd1a and Jmjd2c, as revealed by realtime RT-qPCR (data not shown). The cells were frozen and thawed at different passages, with no significant decrease in their propagation potential and viability, as determined by trypan blue staining. We used 70-80\% confluent DPSCs of passages 3-5 for transfection. They were stained with Hoechst 33258 and no sign of cytoplasmic microorganisms was observed.

We then examined the effect of Olig2 transcription factor in facilitating the glial differentiation of human adult DPSCs. The Olig2 ORF was sequenced before transfection to confirm the right direction, size and sequence of the Olig2 ORF. Random-field counting showed that cell death was significantly increased $(52.64 \pm 5.38 \%$, p < 0.05 ) following treatment with neomycin for 2 days. Thereafter, cell morphology changed from long, spindlelike cells to large, round cell bodies with sprouted processes on about the fifth day, only in those cells transfected with pcDNA-Olig2 plasmid (fig. 2b). Cells that obtained transfection reagent only or plasmid only died
96
Cells Tissues Organs 2014;200:93-103 DOI: $10.1159 / 000381668$
Askari/Yaghoobi/Shamsara/ Esmaeili-Mahani 
Fig. 1. Schematic representation of the differentiation protocol used to generate oligodendrocyte-like cells from human DPSCs.
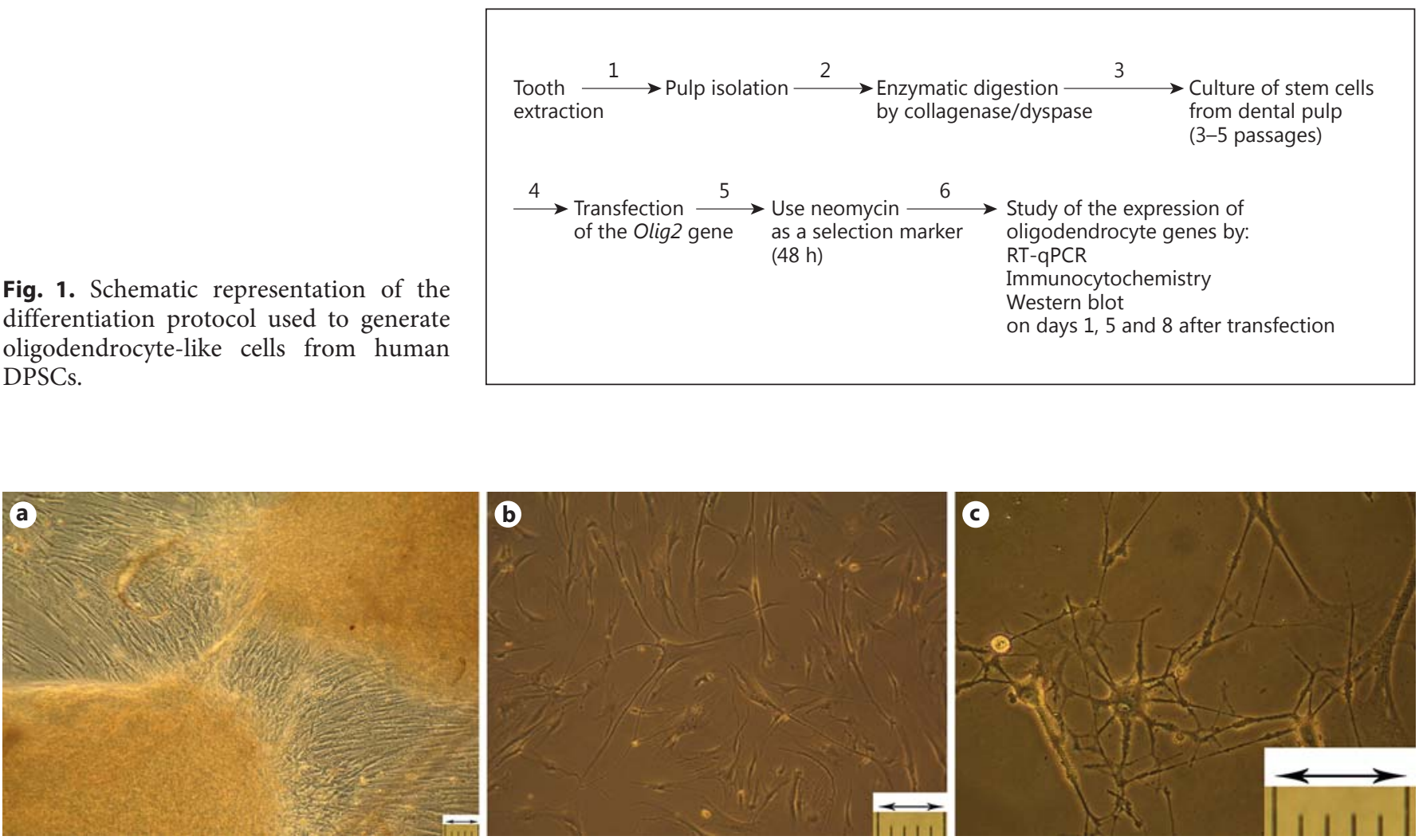

Fig. 2. Differentiation and cellular morphology changes during oligodendroglial-lineage cell differentiation. a Sprouted DPSCs from pulp tissue were observed after 2-3 days. b Cells display im- mature oligodendrocyte (multipolar morphology). c Typical observation of OPCs at day 8 following transfection with Olig2. a-c Scale bar: $50 \mu \mathrm{m}$. following treatment with neomycin. The cells that obtained free backbone of the vector survived and continued their growth for several days without any change in their morphology. The proportion of OPCs increased with time and the cell population had increased about 2 days after the elimination of neomycin from the medium. At day 8, multiple processes arose from the cell bodies. The outgrowth of these processes occurred preferentially towards neighboring cells (fig. 2c). The overall efficiency of gaining OPCs was $<10 \%$ of the total of the cells that were transfected. To provide detailed information and reproducibility, our biological experiments were run in triplicate.

\section{Real-Time RT-qPCR}

Next, the changes in the mRNA expression of oligodendrocyte markers were quantified by real-time RTqPCR assays. DPSCs expressed Nestin and Oct 4 before transfection as confirmed by RT-qPCR. After transfection on the fifth day, DPSCs displayed immature oligo- dendrocytes with multipolar morphology and the expression levels of Nestin and Oct 4 had declined. We also evaluated the OPCs at day 8 when the cells showed an oligodendroglial morphology with multiple branches. The RT-qPCR results demonstrated that in DPSC-derived OPCs, the expression of Oct 4 and Sox 2 was gradually downregulated during the course of differentiation. Additionally, the cells were examined by RT-qPCR at days 1,5 and 8 of differentiation for the expression of markers typical of oligodendrocyte development. The expression of PDGFR $\alpha$, Nestin, NG2 and Olig2 was upregulated at day 5 and downregulated after more differentiation at day 8. Furthermore, DPSC-derived OPCs expressed MBP 8 days after transfection (fig. 3).

In order to trust the differential gene expressions which are detected, it is crucial to validate reference genes in the particular experimental system. Among some candidate reference genes, $\beta$-actin has been considered as a constitutive housekeeping gene for RT-qPCR and is used to normalize changes in specific gene expressions in DPSCs. 


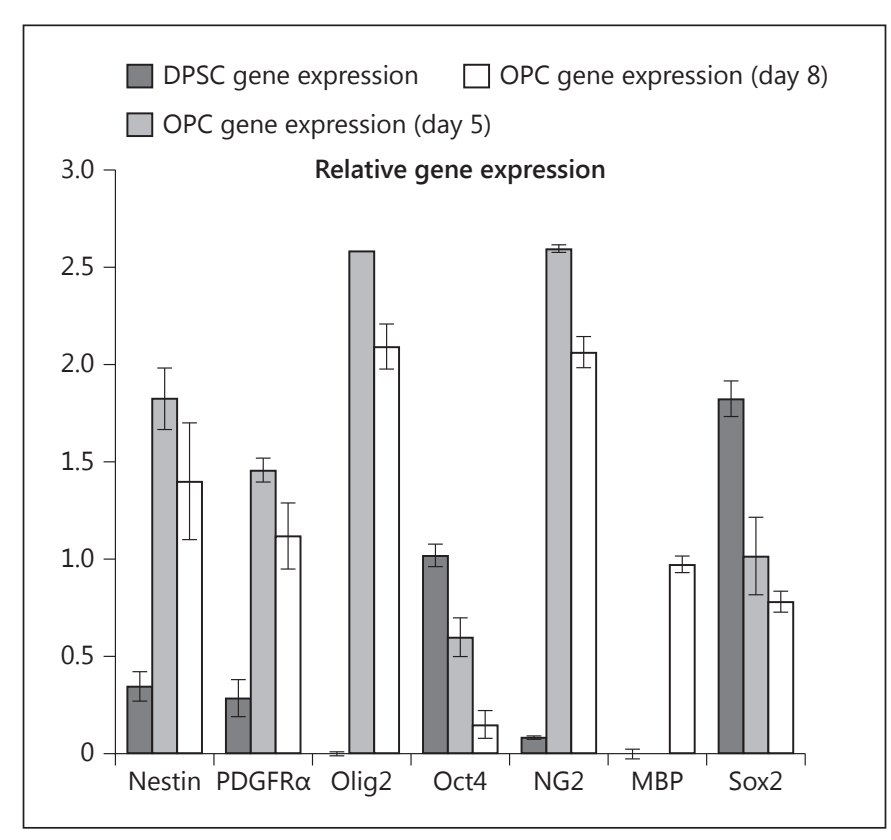

Fig. 3. Quantitative RT-qPCR of PDGFRa, Sox2, Nestin, MBP, NG2, Oct4 and Olig2 transcripts. Quantification was performed in OPs after normalization to $\beta$-actin. Expression levels of PDGFRa, Nestin, MBP, NG2 and Olig2 ( $p<0.05)$ increased significantly in comparison to the control group (DPSCs) 8 days after transfection. Expression levels of Sox 2 and Oct $4(\mathrm{p}<0.05)$ decreased after transfection of Olig2 in comparison to controls (DPSCs).

\section{Immunocytochemistry}

The cells were also evaluated by immunofluorescence staining on days 1, 5 and 8 . Immunostaining for the oligodendrogenic transcription factors Olig2 and GFAP as markers of neuronal precursors and astrocytes, respectively, was performed at day 5 on both differentiated and undifferentiated DPSCs. Whereas no expression of Olig2 and GFAP was detected in the untransfected DPSCs, almost all of the differentiated DPSCs displayed significant levels of Olig2 (95.34 \pm 3.28$)$ and GFAP $(96.41 \pm 2.15$, $\mathrm{p}<0.05$ ). In addition, the membrane epitope typically expressed in OPCs that bind to the monoclonal antibody, A2B5, was also expressed in the DPSC-derived OPCs at day $5(87.69 \pm 1.58)(\mathrm{p}<0.05)$. After 8 days, OPCs expressed MBP which is specifically localized in the myelin sheets of oligodendrocytes $(85.74 \pm 6.14, \mathrm{p}<0.05$; fig. 4$)$.

\section{Western Blot}

The increase in Olig2 after differentiation was also confirmed by Western blot analysis. Olig2 protein which was not expressed before transfection was detected on nitrocellulose membrane containing total protein extracted from the cells 5 days after induction (fig. 5). Band intensities were measured by a densitometer and normalized with $\beta$-actin. In this experiment, the densitometric analysis of the blots was done using Image Quant TL software (GE Healthcare). Western blot strips were examined by reflectance densitometry and the Olig2 antibody response was defined corresponding to the band $(\mathrm{p}<0.05)$. Data are expressed as means \pm SE. Western blots are representative of 3 independent experiments (fig. 5).

\section{Cell Transplantation into the Lysolecithin-Treated Mouse Model of Demyelination}

Typically, the von Frey threshold increased from $0.05 \mathrm{~g}$ (before lysolecithin treatment) to $10.6 \mathrm{~g}$ (after the treatment) target force. It decreased throughout the experiment and had reached $2.49 \mathrm{~g}$ by 6 weeks after the OPC transplant. However, in the groups that received DPSCs and PBS, the threshold reached 9.75 and $10.11 \mathrm{~g}$, respectively. Similarly, thermal withdrawal latencies were raised after lysolecithin treatment of the sciatic nerve; after cell transplantation in the OPC group, they had declined from 31.53 to $21.14 \mathrm{~s}$ by the sixth week, but no reduction was detected in the DPSC and PBS groups. Treadmill exercise revealed that almost none of the lysolecithin-treated mice completed a full $30 \mathrm{~min}$ of treadmill exercise at $12 \mathrm{~m} / \mathrm{min}$; the time actually decreased 1 week after the lysolecithin treatment. At the same time, all mice in the sham group were capable of completing the $30 \mathrm{~min}$ of treadmill exercise without taking rests. Six weeks after cell transplantation, the average times of treadmill exercise in the OPC, DPSC and PBS groups were 14.8, 10.75 and $11.33 \mathrm{~min}$, respectively (fig. 6-8).

The presence of CM-DiI-labeled cells was demonstrated by the observation of red-positive spots in the cross section of sciatic nerve. The results confirmed the viability and presence of the transplanted cells in the sciatic nerve 6 weeks after transplantation (fig. 9).

Sections of nerve from the region of lysolecithin application were dissected and tumor formation in the transplanted animals was assessed by HE staining. There was no sign of tumor formation in the sections of sciatic nerve. Nerve morphology was examined in animals that had previously had focal demyelination in their sciatic nerves. We applied lysolecithin topically to the sciatic nerve, producing a focal demyelination. Observation of the sciatic nerve sections under light microscopy showed that the nerves were demyelinated after lysolecithin treatment in the PBS and DPSC groups, and many of the remaining myelinated fibers had myelin of reduced thickness. Remyelination was observed in the OPC group (fig. 10).
Askari/Yaghoobi/Shamsara/ Esmaeili-Mahani 

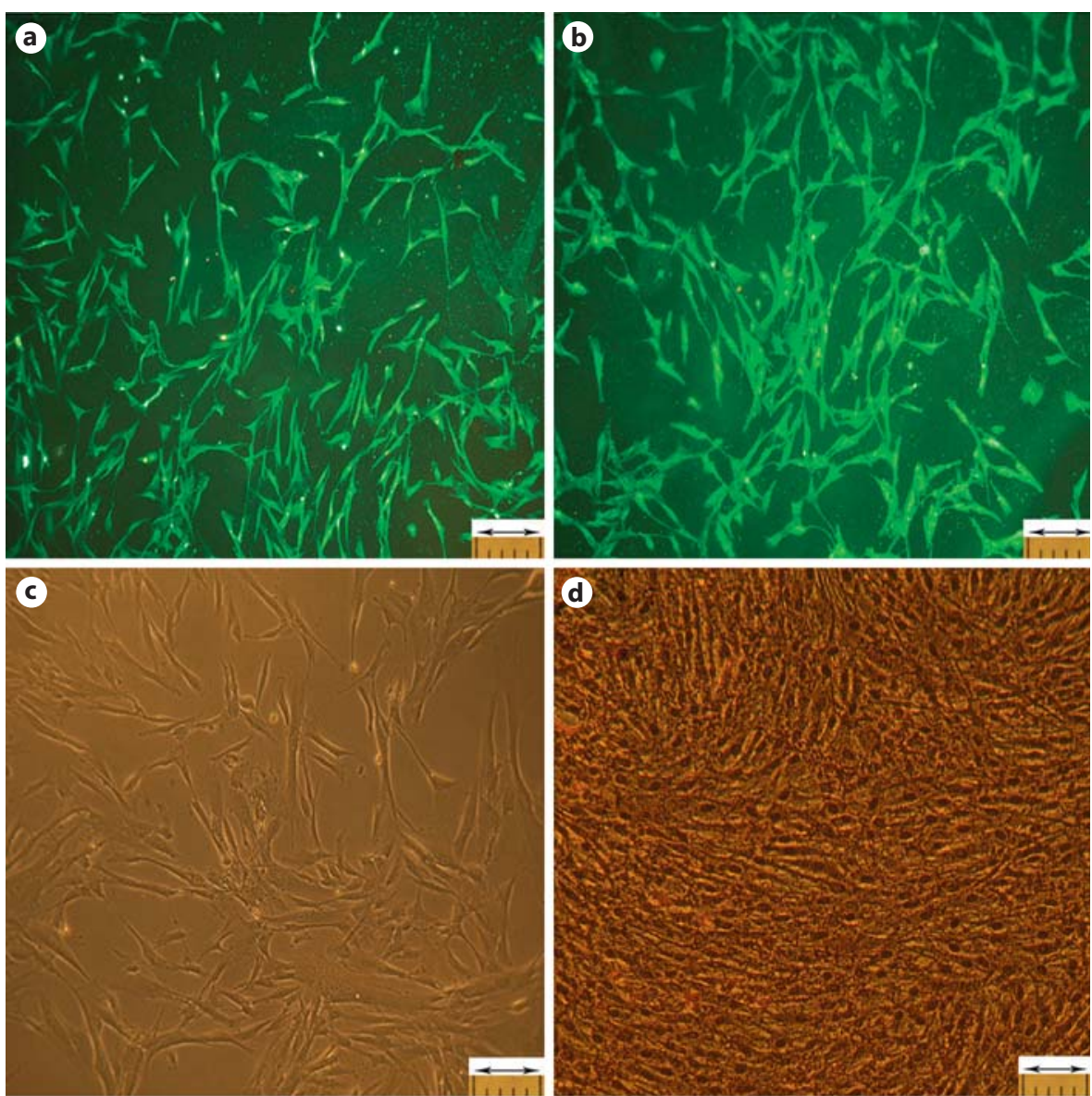

Fig. 4. Immunocytochemistry staining of OPCs derived from DPSCs. The representative pictures of Olig2, GFAP, A2B5, $\mathrm{MBP}$ expression in DPSC-derived OPCs. a Immunofluorescence staining of A2B5 in OPCs (day 5). b Immunofluorescence staining of MBP in OPCs (day 8). c Negative control of Olig2 in DPSCs. d Olig2 expression in OPCs (day 5). e Negative control of GFAP in DPSCs. f GFAP expression in OPCs (day 5). a-f Scale bar: $50 \mu \mathrm{m}$.
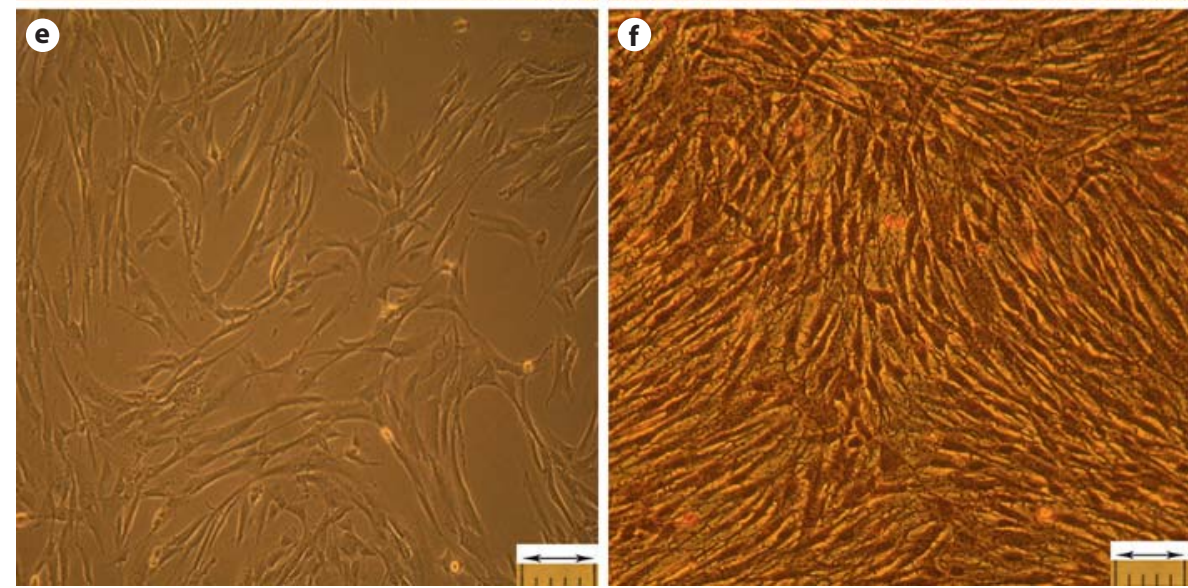

The sciatic nerve sections that were examined revealed changes consisting of foci of myelin aggregation, vacuolated foci and nerve cell proliferation. Vacuolated foci were less common in the OPCs transplantation group. In the PBS and DPSC groups, there were larger vacuolated foci of myelin aggregation.

Olig2 Differentiates DPSCs into

Oligodendrocyte Progenitors

\section{Discussion}

Our results show that the overexpression of Olig2 in DPSCs by gene transfection can promote their differentiation into oligodendrocyte progenitors. Many studies have demonstrated that the transcription factors Olig1 and Olig2 are critically involved in the formation of oli- 
Fig. 5. a Western blot analysis of Olig2 protein expression in transfected DPSCs compared with untransfected DPSCs. b The Western blot bands were quantified by densitometry after normalization to $\beta$-actin protein expression. Data are means \pm SD of triplicate experiments. ${ }^{*} \mathrm{p}<0.05$.

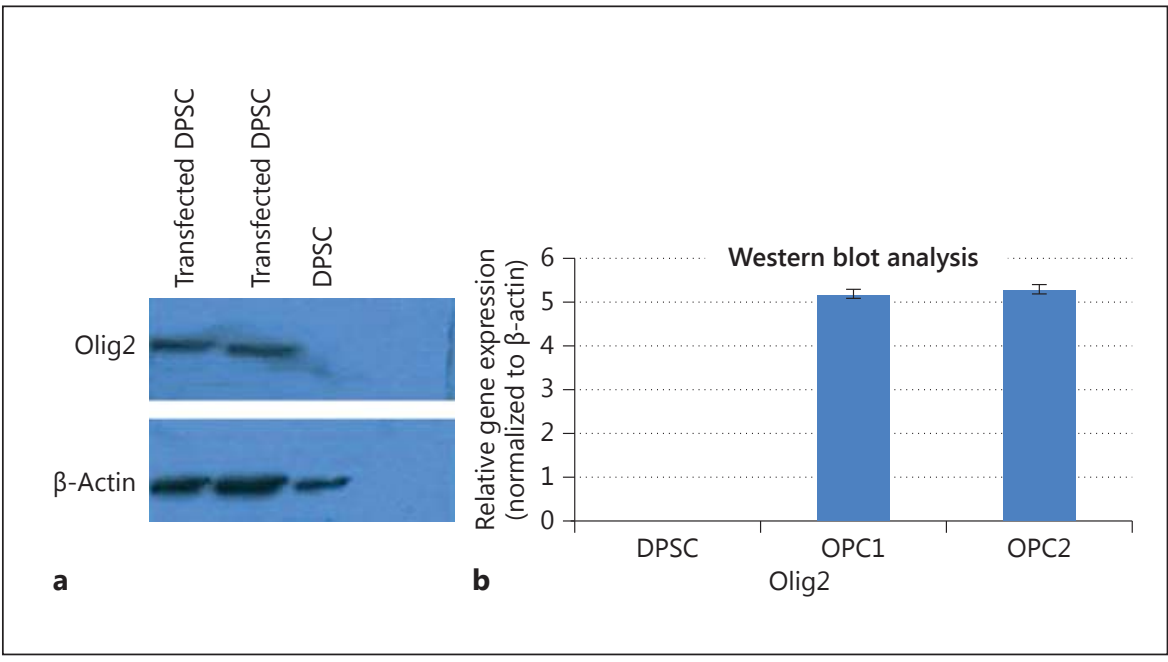

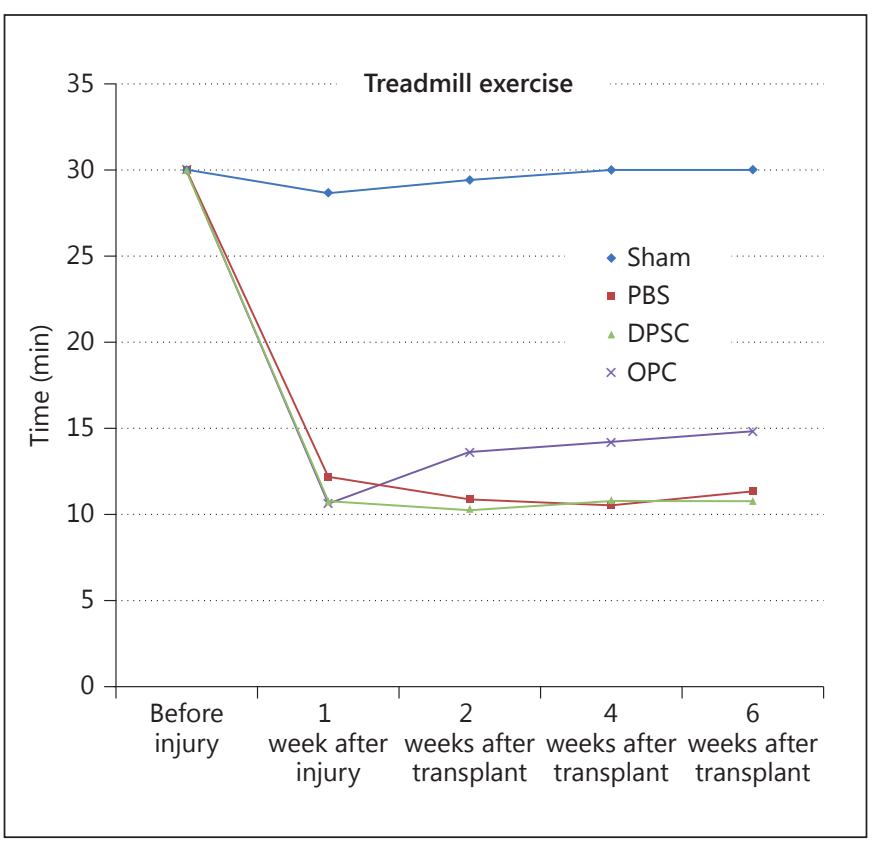

Fig. 6. Mechanical sensitization using treadmill exercise from before injury to 6 weeks after cell transplantation.

godendrocytes and in myelination during embryogenesis. Zhang et al. [2005] showed that cotransfection of Olig2 plus Nkx2.2 could induce oligodendrocyte differentiation in human olfactory epithelial-derived progenitor cells. Copray et al. [2006] also showed that Olig2 transfection can be an efficient way to induce differentiation of neural stem cells into functional oligodendrocytes.

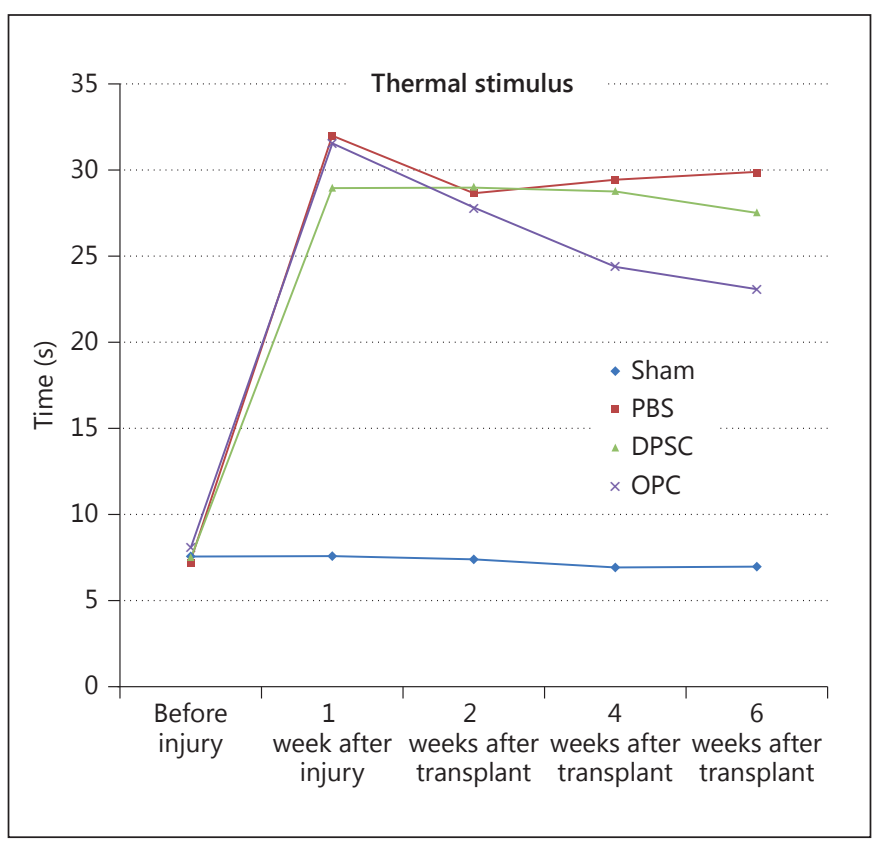

Fig. 7. Touch sensitization using thermal sensitivity from before injury to 6 weeks after cell transplantation.

Dental pulp is made of both mesenchymal and ectodermic components containing neural crest cells that display multipotential and plasticity capability. They display a high proliferation rate, easy accessibility and the capacity for multidifferentiation. DPSCs express many pluripotency markers including Nanog, Oct4, Nucleostemin, Slain-1, Jmjd1, Jmjd2 and cyclin D1 [Kerkis et al., 2007; Ebrahimi et al., 2011]. We also observed mRNA 


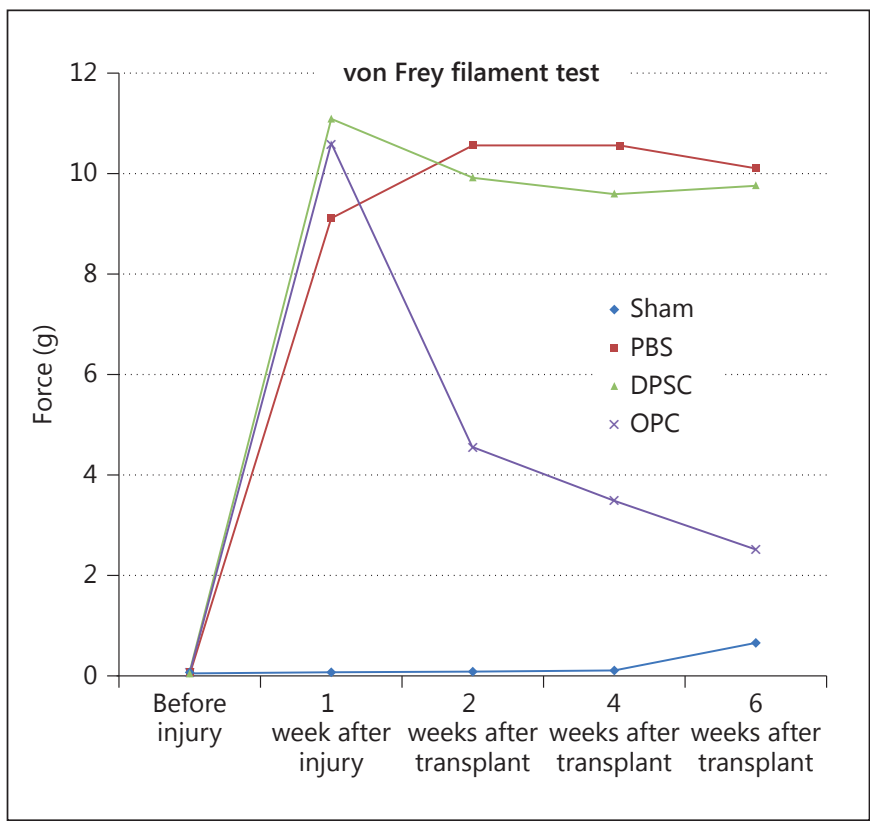

Fig. 8. Touch sensitization using von Frey filaments from before injury to 6 weeks after cell transplantation.

expression of some markers (Oct4 and Sox2) in the isolated stem cells for this research. However, in order to be brief and to avoid repetition, the data were not shown. DPSCs also show potential in regenerative medicine because of their capacity to differentiate into several different types of cells: odontoblasts, osteoblasts, melanocytes, neurons, chondrocytes and adipocytes [Tatullo et al., 2014].

In this work, isolated DPSCs expressed Sox 2 and Oct 4 as the markers of pluripotency. These two markers were gradually downregulated on days 5-8 after transfection. Hence, the DPSCs started to differentiate by decreasing the markers of pluripotency in this way. On the other hand, the expression of PDGFRa, Nestin, NG2 and Olig2 increased on the fifth day after DPSC-derived OPCs differentiated into preoligodendrocytes. Nevertheless, when they continued to differentiate into mature oligodendrocytes, the expression of these genes was downregulated by day 8 . Finally, the cells expressed MBP on day 8. Our data also showed the expression of PDGFRa in this manner.

Olig2 and GFAP immunoreactivity was observed in DPSC-derived OPCs. They were positive for A2B5, in addition to Olig2 and GFAP, 5 days after transfection. As the cells continued to differentiate more into oligodendrocytes, they were positively stained for MBP. We ob-
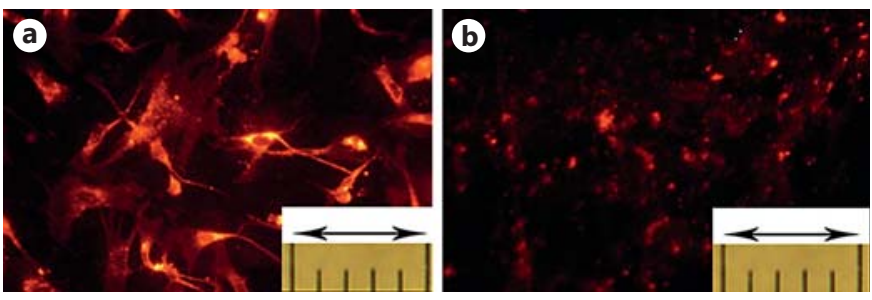

Fig. 9. a OPCs labeled with CM-DiI before transplantation. b Cross sections of the sciatic nerve 6 weeks after the transplantation of OPCs labeled with CM-DiI showing as red spots in the sciatic nerve. a, b Scale bar: $50 \mu \mathrm{m}$.
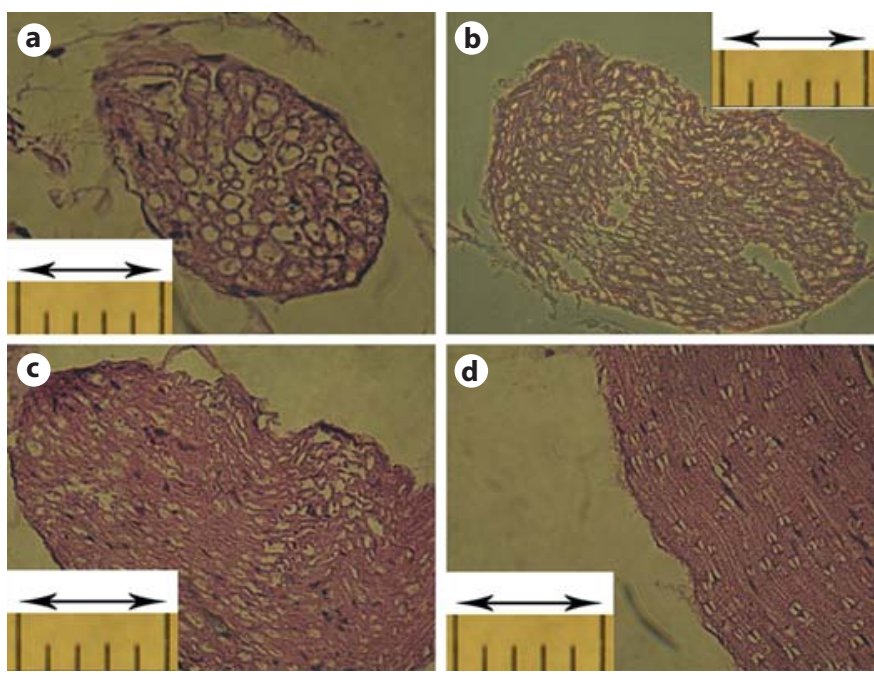

Fig. 10. Effects of lysolecithin treatment and myelination state of the sciatic nerve after 6 weeks in HE-stained sections of sciatic nerves in the following groups: DPSCs (a), PBS (b), DPSC-derived OPCs (c) and the sham group (d). a-d Scale bar: $50 \mu \mathrm{m}$.

served that the OPC-like cells derived from DPSCs expressed transcription factor Olig2 as a specific oligodendrocyte marker. Moreover, we continued to culture adherent OPCs for several passages (results not shown). Our results indicate that DPSC-derived OPCs can differentiate into more mature oligodendrocytes. Other reported data support our findings. Zhang et al. [2010] demonstrated the transdifferentiation of mesenchymal stem cells into oligodendrocytes. They showed that human Wharton's jelly-derived mesenchymal stromal cells can be differentiated into oligodendrocyte progenitorlike cells capable of secreting nerve growth factors and promoting neurite outgrowth. Peng et al. [2011] also reported that Wharton's jelly-derived mesenchymal stem 
cells differentiated into cells which were Schwann-like in terms of their morphologic features, phenotype and function. In addition, Ebrahimi-Barough et al. [2013] speculated that exposing endometrial adult stem cells to specific signaling molecules and neuronal condition media can differentiate these cells into OPCs.

We showed that the transplantation of OPCs led to functional recovery of the injured sciatic nerve. This was documented by the von Frey filament test and the thermal withdrawal latencies test as well as the walking behavior measured on the treadmill. The results of the tests revealed that there were statistically significant differences between the control and experimental groups (i.e. the sham, PBS, DPSC and OPC groups). Based on the manufacturer's protocol (North Coast Medical Inc.), there are five separate parts to the plantar foot threshold of the von Frey filament test: normal, diminished light sensation, diminished protective sensation, loss of protective sensation and deep pressure sensation only. One week after the lysolecithin treatment, sensitivity decreased from 'normal' on the threshold-of-touch test evaluator chart to 'diminished protective sensation'. However, 4 weeks after cell transplantation, it became 'diminished light sensation'. After 6 weeks, it reached the edge of the part on the chart indicating 'normal' but remained in the 'diminished light sensation' part. Foci of myelin aggregation were observed in association with these processes in the OPC group during these weeks in comparison to the PBS and DPSC groups. In the PBS and DPSC groups, there was some evidence of sciatic nerve fiber degeneration. The treadmill exercise that we used in our experiment did not exhaust the animals; they achieved $30 \mathrm{~min}$ of treadmill running at a speed of $12 \mathrm{~m} / \mathrm{min}$. The presence and function of the CM-DiI-labeled cells were confirmed by the viability of the transplanted cells in the sciatic nerves 6 weeks after the cell transplantation. DPSC-derived OPC transplantation showed increased remyelinating axons. The engrafted OPCs integrated into the host tissue after cell transplantation. Functional changes to the sciatic nerve were recorded with behavioral tests. It was also shown that DPSC-derived OPCs promoted recovery in the transplanted mice compared to the PBS, DPSC and control groups. This improvement was observed particularly in the second week after cell transplantation, which indicates the short-term effectiveness of OPC transplantation. Transplantation of stem cells from the bone marrow and adipose tissue as well as embryonic and neuronal stem cells into the transected sciatic nerve showed clinical improvement, inducing vigorous nerve regeneration accompanied by myelin synthesis [Ribeiro et al., 2013]. Sev- eral investigators have demonstrated that Schwann cells and bone marrow stromal cells can repair the sciatic nerve injuries [Braga-Silva et al., 2006; Chen et al., 2007; Gu et al., 2011]. There is thus good evidence to support the hypothesis that the transplantation of OPCs may repair more effectively peripheral nerve injuries.

Marconi et al. [2012] reported that an intravenous administration of adipose-derived mesenchymal stem cells 1 week after sciatic nerve injury in a mouse model of acute axonal damage significantly accelerated the functional recovery. In comparison to our study, previous studies aiming at restoring sciatic nerve function after injury have employed scaffolds and artificial nerve conduits. The limitation of these methods of administration is the additional trauma induced in reaching the injection site as well as the difficulty in treating the sites of injury [Deumens et al., 2010]. Numerous surgical techniques are performed each year for peripheral nerve regeneration. Sciatic nerve injury resulting in peripheral nerve traumas requires a graft to remove the defect. Autologous nerve grafting is the method for bridging peripheral nerve defects, but has the disadvantage of the sacrifice of a functional nerve. In recent years, cell transplantation has become a focus of attention, and reliable outcomes have been achieved in the regeneration of the sciatic nerve [di Summa et al., 2010]. On the other hand, adult tissues are a valuable source of stem cells, and the autologous transplantation of DPSCs can be used to treat a variety of traumas without any rejection.

To the best of our knowledge, our study describes a new approach to the ability of human DPSCs as a source of mesenchymal stem cells to differentiate into oligodendrocyte progenitors with characteristic OPC morphology, expressing markers of OPC phenotype and the potential to repair demyelinated peripheral nerves.

Collectively, our results suggest that the transplantation of OPCs has the potential to generate the functional recovery of injured sciatic nerves in mice. Evaluation of the behavioral tests revealed that the OPC transplantation resulted in a greater functional improvement in the injured nerve compared to the other groups. Our findings revealed the use of DPSC-derived OPCs for the clinical repair of the injured peripheral nerves. They present the possibility of a novel form of cell therapy as a treatment for patients suffering from different peripheral nerve diseases with degenerative pathogenesis from traumatic nerve injuries. 


\section{Acknowledgments}

This work was conducted in the Graduate University of Advanced Technology and was supported financially by grant No. 91003151 from the Iran National Science Foundation. We thank Dr. Ali Shafieipour for technical assistance.

\section{Disclosure Statement}

The authors indicate no potential conflicts of interest.

\section{References}

Braga-Silva, J., D. Gehlen, et al (2006) Bone marrow stem cells and platelet-rich plasma effects on nervous regeneration and functional recovery in an acute defect model of rats' peripheral nerve. Acta Ortop Bras 14:273-275.

Cai, J., Y. Qi, et al (2005) Generation of oligodendrocyte precursor cells from mouse dorsal spinal cord independent of $\mathrm{Nkx} 6$ regulation and signaling. Neuron 45: 41-53.

Chen, C.-J., Y.-C. Ou, et al (2007) Transplantation of bone marrow stromal cells for peripheral nerve repair. Exp Neurol 204: 443-453.

-Copray, S., V. Balasubramaniyan, et al (2006) Olig2 overexpression induces the in vitro differentiation of neural stem cells into mature oligodendrocytes. Stem Cells 24: 1001-1010.

Deumens, R., A. Bozkurt, et al (2010) Repairing injured peripheral nerves: bridging the gap. Prog Neurobiol 92: 245-276.

di Summa, P.G., P.J. Kingham, et al (2010) Adipose-derived stem cells enhance peripheral nerve regeneration. J Plast Reconstr Aesthet Surg 63: 1544-1552.

Ebrahimi, B., M. Yaghoobi, et al (2011) Human dental pulp stem cells express many pluripotency regulators and differentiate into neuronal cells. Neural Regen Res 6: 2666-2672.

-Ebrahimi-Barough, S., H.M. Kouchesfahani, et al (2013) Differentiation of human endometrial stromal cells into oligodendrocyte progenitor cells (OPCs). J Mol Neurosci 51: 265-273.

-Grinspan, J. (2002) Cells and signaling in oligodendrocyte development. J Neuropathol Exp Neurol 61: 297-306.

Gu, X., F. Ding, et al (2011) Construction of tissue engineered nerve grafts and their application in peripheral nerve regeneration. Progr Neurobiol 93: 204-230.
Hall, S.M., N. Gregson (1971) The in vivo and ultrastructural effects of injection of lysophosphatidyl choline into myelinated peripheral nerve fibres of the adult mouse. J Cell Sci 9: 769-789.

Hargreaves, K., R. Dubner, et al (1988) A new and sensitive method for measuring thermal nociception in cutaneous hyperalgesia. Pain 32 : 77-88.

Huang, A.H.C., Y.K. Chen, et al (2008) Isolation and characterization of dental pulp stem cells from a supernumerary tooth. J Oral Pathol Med 37: 571-574.

Kerkis, I., A. Kerkis, et al (2007) Isolation and characterization of a population of immature dental pulp stem cells expressing OCT-4 and other embryonic stem cell markers. Cells Tis sues Organs 184: 105-116.

Liu, Y., M.S. Rao (2004) Glial progenitors in the CNS and possible lineage relationships among them. Biol Cell 96: 279-290.

Lu, Q.R., D.-i. Yuk, et al (2000) Sonic hedgehogregulated oligodendrocyte lineage genes encoding bHLH proteins in the mammalian central nervous system. Neuron 25: 317-329.

Marconi, S., G. Castiglione, et al (2012) Human adipose-derived mesenchymal stem cells systemically injected promote peripheral nerve regeneration in the mouse model of sciatic crush. Tissue Eng Part A 18: 1264-1272.

$\checkmark$ Peng, J., Y. Wang, et al (2011) Human umbilical cord Wharton's jelly-derived mesenchymal stem cells differentiate into a Schwann-cell phenotype and promote neurite outgrowth in vitro. Brain Res Bull 84: 235-243.
Ribeiro, J., A. Gartner, T. Pereira, R. Gomes, M.A. Lopes, C. Goncalves, A.C. Maurício (2013) Perspectives of employing mesenchymal stem cells from the Wharton's jelly of the umbilical cord for peripheral nerve repair. Int Rev Neurobiol 108: 79-120.

Rogister, B., T. Ben-Hur, et al (1999) From neural stem cells to myelinating oligodendrocytes Mol Cell Neurosci 14: 287-300.

Rowitch, D.H., Q.R. Lu, et al (2002) An 'oligarchy' rules neural development. Trends Neurosci 25: 417-422.

Ruijter, J., C. Ramakers, et al (2009) Amplification efficiency: linking baseline and bias in the analysis of quantitative PCR data. Nucleic Acids Res 37: e45-e45.

Tatullo, M., M. Marrelli, K.M. Shakesheff, L.J. White (2014) Dental pulp stem cells: function, isolation and applications in regenerative medicine. J Tissue Eng Regen Med, Epub ahead of print.

Vallstedt, A., J.M. Klos, et al (2005) Multiple dorsoventral origins of oligodendrocyte generation in the spinal cord and hindbrain. Neuron 45: 55-67.

Yalvac, M.E., A.A. Rizvanov, et al (2009) Potential role of dental stem cells in the cellular therapy of cerebral ischemia. Curr Pharm Des 15: 3908-3916.

Zhang, H.-T., J. Fan, et al (2010) Human Wharton's jelly cells can be induced to differentiate into growth factor-secreting oligodendrocyte progenitor-like cells. Differentiation 79: 1520.

Zhang, X., J. Cai, et al (2005) Induction of oligodendrocytes from adult human olfactory epithelial-derived progenitors by transcription factors. Stem Cells 23: 442-453.
Olig2 Differentiates DPSCs into

Oligodendrocyte Progenitors
Cells Tissues Organs 2014;200:93-103 DOI: $10.1159 / 000381668$ 